1 Hacettepe Journal of Mathematics and Statistics

$\bigcap$ Volume 47 (3) (2018), 513-519

\title{
On super edge-magic deficiency of certain Toeplitz graphs
}

\author{
Ali Ahmad*, Muhammad Faisal Nadeem ${ }^{\dagger}$ and Ashok Gupta ${ }^{\ddagger \S}$
}

\begin{abstract}
A graph $G$ is called edge-magic if there exists a bijective function $\phi: V(G) \cup E(G) \rightarrow\{1,2, \ldots,|V(G)|+|E(G)|\}$ such that $\phi(x)+$ $\phi(x y)+\phi(y)=c(\phi)$ is a constant for every edge $x y \in E(G)$, called the valence of $\phi$. Moreover, $G$ is said to be super edge-magic if $\phi(V(G))=\{1,2, \ldots,|V(G)|\}$. The super edge-magic deficiency of a graph $G$, denoted by $\mu_{s}(G)$, is the minimum nonnegative integer $n$ such that $G \cup n K_{1}$, has a super edge-magic labeling, if such integer does not exist we define $\mu_{s}(G)$ to be $+\infty$. In this paper, we study the super edge-magic deficiency of some Toeplitz graphs.
\end{abstract}

Keywords: edge-magic, super edge-magic, Toeplitz graphs

2000 AMS Classification: $05 \mathrm{C} 78$

Received : 02.01.2017 Accepted : 11.05.2017 Doi : 10.15672/HJMS.2017.465

\section{Introduction and preliminary results}

In this paper, we consider only finite, simple and undirected graphs $G=(V, E)$. We denote the vertex set and edge set of a graph $G$ by $V(G)$ and $E(G)$ respectively, where $|V(G)|=p$ and $|E(G)|=q$. An edge-magic labeling of a graph $G$ is a bijection $\phi: V(G) \cup E(G) \rightarrow\{1,2, \ldots, p+q\}$, where there exists a constant $c(\phi)$ such that $f(x)+f(x y)+f(y)=c(\phi)$, for every edge $x y \in E(G), c(\phi)$ is called the valence of the graph and a graph with an edge-magic labeling is called edge-magic. An edge-magic labeling $\phi$ is called a super edge-magic labeling of $G$, and $G$ is said to be super edge-magic if $\phi(V(G))=\{1,2, \ldots, p\}$. It is with to mention that super edge-magic labelings where independently defined by Acharia and Hegde in [1].

${ }^{*}$ College of Computer Science \& Information Systems, Jazan University, Jazan, KSA.

Email : ahmadsms@gmail.com

$\dagger$ Department of Mathematics, Comsat Institute of Information Technology, Lahore, Pakistan. Email : m.fayssal@gmail.com

${ }_{\text {}}$ College of Computer Science \& Information Systems, Jazan University, Jazan, KSA.,

Email : kgupta.ashok@gmail.com

$\S$ Corresponding Author. 
In [9], Kotzig and Rosa proved that for any graph $G$ there exists an edge-magic graph $H$ such that $H \cong G \cup n K_{1}$ for some nonnegative integer $n$. This fact leads to the concept of edge-magic deficiency of a graph $G$, which is the minimum nonnegative integer $n$ such that $G \cup n K_{1}$ is edge-magic and it is denoted by $\mu(G)$. In particular,

$$
\mu(G)=\min \left\{n \geq 0: G \cup n K_{1} \text { is edge-magic }\right\} .
$$

In the same paper, Kotzig and Rosa gave an upper bound for the edge-magic deficiency of a graph $G$ with $n$ vertices, $\mu(G) \leq F_{n+2}-2-n-\frac{1}{2} n(n-1)$, where $F_{n}$ is the $n^{t h}$ Fibonacci number. Motivated by Kotzig and Rosa's concept of edge-magic deficiency, Figueroa-Centeno et al. [7] defined a similar concept for super edge-magic labelings. The super edge-magic deficiency of a graph $G$, which is denoted by $\mu_{s}(G)$, is the minimum nonnegative integer $n$ such that $G \cup n K_{1}$ has a super edge-magic labeling or $+\infty$ if there exists no such $n$.

Let $M(G)=\left\{n \geq 0: G \cup n K_{1}\right.$ is a super edge-magic graph $\}$, then

$$
\mu_{s}(G)=\left\{\begin{array}{lll}
\min M(G), & \text { if } & M(G) \neq \phi \\
+\infty, & \text { if } & M(G)=\phi .
\end{array}\right.
$$

As a consequence of the above two definitions, for every graph $G, \mu(G) \leq \mu_{s}(G)$. In [7,8], Figueroa-Centeno et al. provided the exact values for the super edge-magic deficiency of several classes of graphs, such as cycles, complete graphs, 2-regular graphs, and complete bipartite graphs $K_{2, m}$. They also proved that all forests have finite deficiency. In [10], A. A. G. Ngurah et al. proved some upper bounds for the super edge-magic deficiency of fans, double fans, and wheels. In [2], A. Ahmad et al. found the super edge-magic deficiency of some families related to ladder graphs. In this paper, we discuss the super edge-magic deficiency of some families of Toeplitz graphs.

In proving the results, we frequently use the following lemmas.

1.1. Lemma. [6] A graph $G$ with $p$ vertices and $q$ edges is super edge-magic if and only if there exists a bijective function $\psi: V(G) \rightarrow\{1,2, \cdots, p\}$ such that the set $S=$ $\{\phi(x)+\phi(y): x y \in E(G)\}$ consists of $q$ consecutive integers. In such a case, $\psi$ extends to a super edge-magic total labeling of $G$ with magic constant $c(\psi)=p+q+s$, where $s=\min (S)$ and

$$
S=\{c(\psi)-(p+1), c(\psi)-(p+2), \ldots, c(\psi)-(p+q)\} .
$$

1.2. Lemma. [5] If a graph $G$ with $p$ vertices and $q$ edges is super edge-magic, then $q \leq 2 p-3$.

\section{Results and Discussion}

A simple undirected graph $T$ of order $p$ is called a Toeplitz graph if its adjacency matrix $A(T)$ is Toeplitz. A Toeplitz matrix $A(T)=\left(a_{i, j}\right)$, is a $(p \times p)$ symmetric matrix which has constant values along all diagonals parallel to the main diagonal, i.e. $a_{i, j}=a_{i+1, j+1}$ for each $i, j=1,2, \ldots, p-1$. The $p$ distinct diagonals of a $(p \times p)$ symmetric Toeplitz adjacency matrix will be labeled $0,1,2, \ldots, p-1$. Diagonal 0 is the main diagonal and it contains only zeros, i.e. $a_{i i}=0$ for all $i=1,2, \ldots, p$ so that there are no loops in the Toeplitz graph. A Toeplitz graph $T$ is uniquely defined by the first row of $A(T)$, a $(0-1)$ sequence. Let $t_{1}, t_{2}, \ldots, t_{k}$ be the diagonals containing ones, $0<t_{1}<t_{2}<\cdots<t_{k}<$ $p$. Then, the corresponding Toeplitz graph will be denoted by $T_{p}\left\langle t_{1}, \ldots, t_{k}\right\rangle$. That is, $T_{p}\left\langle t_{1}, \ldots, t_{k}\right\rangle$ is the graph with the vertex set $V(T)=\left\{v_{i}: i=1,2, \ldots, p\right\}$ in which two vertices $u, v$ of $T$ being connected by an edge if and only if $|u-v| \in\left\{t_{1}, t_{2}, \ldots, t_{k}\right\}$. If $t_{j}, j=1,2, \ldots, k$, is a diagonal containing ones then the diagonal elements $a_{i, t_{j}+i}$, $i=1,2, \ldots, p-t_{j}$, determining the edges $v_{i} v_{t_{j}+i}$ in the Toeplitz graph. Thus the edge 
set is $E(T)=\bigcup_{j=1}^{k}\left\{v_{i} v_{t_{j}+i}: i=1,2, \ldots, p-t_{j}\right\},|V(T)|=p$ and $|E(T)|=p k-\sum_{j=1}^{k} t_{j}$. In [4] Bača et al. determined super edge-antimagic total labeling of Toeplitz graphs and Ahmad et al. [3] determined the edge irregularity strength of Toeplitz graphs. A Toeplitz graph is not necessarily connected, see Figure 1.

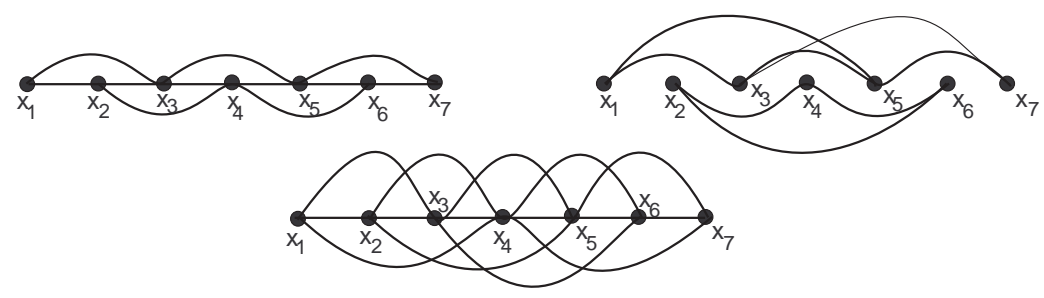

Figure 1. Toeplitz graphs $T_{7}\langle 1,2\rangle, T_{7}\langle 2,4\rangle$ and $T_{7}\langle 1,2,3\rangle$

The following Theorem shows a lower bound for the super edge-magic deficiency for the Toeplitz graph $T_{p}\left\langle t_{1}, \ldots, t_{k}\right\rangle$.

2.1. Theorem. The lower bound for the super edge-magic deficiency for Toeplitz graph $T_{p}\left\langle t_{1}, \ldots, t_{k}\right\rangle$ is $\frac{p(k-2)+3-\sum_{j=1}^{k} t_{j}}{2}$.

Proof. The vertex set of $T_{p}\left\langle t_{1}, \ldots, t_{k}\right\rangle$ is $V\left(T_{p}\left\langle t_{1}, \ldots, t_{k}\right\rangle\right)=\left\{v_{i}: i=1,2, \ldots, p\right\}$ and the edge set of $T_{p}\left\langle t_{1}, \ldots, t_{k}\right\rangle$ is $q=E\left(T_{p}\left\langle t_{1}, \ldots, t_{k}\right\rangle\right)=\bigcup_{j=1}^{k}\left\{v_{i} v_{t_{j}+i}: i=1,2, \ldots, p-t_{j}\right\}$.

By Lemma 1.2, we know that the size of any super edge-magic graph is bounded above by two times its order minus 3. Now we know that for the Toeplitz graph $T_{p}\left\langle t_{1}, \ldots, t_{k}\right\rangle$, $p=n$ and $q=p k-\sum_{j=1}^{k} t_{j}$, which implies that we need $x \in \mathbb{N}$ such that $p k-\sum_{j=1}^{k} t_{j} \leq$ $2(p+x)-3$ or $p(k-2)+3-\sum_{j=1}^{k} t_{j} \leq 2 x$. This implies that we need at least $\frac{p(k-2)+3-\sum_{j=1}^{k} t_{j}}{2}$ isolated vertices.

In [4], Bača et al. found the super edge-antimagic total labeling of $T_{p}\langle 1,2\rangle$. In the next theorem, we showed that the super edge-magic deficiency of Toeplitz graph $T_{p}\langle 1, t\rangle$ is zero for $t \geq 4$ even.

2.2. Theorem. Let Toeplitz graph $T_{p}\langle 1, t\rangle$ with $p$ vertices, $q$ edges and $t \geq 4$ even admits a super edge-magic labeling i.e $\mu_{s}\left(T_{p}\langle 1, t\rangle\right)=0$

Proof. Let $x_{1}, x_{2}, \ldots, x_{p}$ be the vertex sequence of $T_{p}\langle 1, t\rangle$. The vertex set and edge set of $T_{p}\langle 1, t\rangle$ are defined as:

$$
\begin{gathered}
V\left(T_{p}\langle 1, t\rangle\right)=\left\{x_{i}: 1 \leq i \leq p\right\} . \\
E\left(T_{p}\langle 1, t\rangle\right)=\left\{x_{i} x_{i+1}: 1 \leq i \leq p-1\right\} \cup\left\{x_{i} x_{t+i}: 1 \leq i \leq p-t\right\} .
\end{gathered}
$$


We define the labeling for $T_{p}\langle 1, t\rangle$ as follows:

Case 1: When $p \equiv 0(\bmod 2)$

$$
\phi\left(x_{i}\right)= \begin{cases}\frac{i+1}{2}, & \text { if } 1 \leq i \leq t+1, \text { and } i \equiv 1(\bmod 2) \\ i-\frac{t}{2}, & \text { if } t+3 \leq i \leq p-1, \text { and } i \equiv 1(\bmod 2) \\ i+\frac{t}{2}, & \text { if } 2 \leq i<p-t+2, \text { and } i \equiv 0(\bmod 2) \\ i+\frac{a}{2}, & \text { if } p-t+2 \leq i \leq p, i=p-a, \\ & \text { where } 0 \leq a \leq t-2, \text { and } a, i \text { are even }\end{cases}
$$

Case 2: When $p \equiv 1(\bmod 2)$

$$
\phi\left(x_{i}\right)= \begin{cases}\frac{i+1}{2}, & \text { if } 1 \leq i \leq t+1, \text { and } i \equiv 1(\bmod 2) \\ i-\frac{t}{2}, & \text { if } t+3 \leq i \leq p, \text { and } i \equiv 1(\bmod 2) \\ i+\frac{t}{2}, & \text { if } 2 \leq i \leq p-t+2, \text { and } i \equiv 0(\bmod 2) \\ i+\frac{a+1}{2}, & \text { if } p-t+2 \leq i \leq p, i=p-a, \\ & \text { where } 1 \leq a \leq t-3, \text { and } a \text { odd }, i \text { even }\end{cases}
$$

The set of all edge-sums generated by the above formula forms $q$ consecutive integer sequences. Therefore by using Lemma 1.1, $\phi$ can be extended to a super edge-magic labeling. Since there is no isolated vertex, it follows that $\mu_{s}\left(T_{p}\langle 1, t\rangle\right)=0$. Which completes the proof.

2.3. Open Problem. Consider the graph $T_{p}\langle 1, t\rangle$ with $t$ odd. Show that either $T_{p}\langle 1, t\rangle$ is super edge-magic or calculate its super edge-magic deficiency.

The following theorem shows an upper bound for the super edge-magic deficiency of $T_{p}\langle 1,2,3\rangle$ for $p \geq 5$.

2.4. Theorem. Consider the Toeplitz graph $T_{p}\langle 1,2,3\rangle$ with $p$ vertices and $q$ edges. The super edge-magic deficiency for $T_{p}\langle 1,2,3\rangle$ is upper bounded by

$$
\mu_{s}\left(T_{p}\langle 1,2,3\rangle\right) \leq \begin{cases}\frac{p-1}{2}, & \text { if } p \equiv 1 \quad(\bmod 4) \\ \left\lceil\frac{p-3}{2}\right\rceil, & \text { otherwise }\end{cases}
$$

Proof. Let $x_{1}, x_{2}, \ldots, x_{p}$ be the vertex sequence of $T_{p}\langle 1,2,3\rangle$ and the edges of $T_{p}\langle 1,2,3\rangle$ are $\left\{x_{i} x_{i+1}: 1 \leq i \leq p-1\right\} \cup\left\{x_{i} x_{i+2}: 1 \leq i \leq p-2\right\} \cup\left\{x_{i} x_{i+3}: 1 \leq i \leq p-3\right\}$.

Let $p$ be a nonnegative integer. According to Lemma 1.1 it is sufficient to prove that there exists a vertex labeling with the property that the edge-sums under this labeling are consecutive integers. It is easy to see that the following labelings $\phi_{1}: V\left(T_{p}\langle 1,2,3\rangle \cup\right.$ $\left.\left(\frac{p-1}{2}\right) K_{1}\right) \rightarrow\left\{1,2, \ldots,\left|V\left(T_{p}\langle 1,2,3\rangle\right)\right|+\frac{p-1}{2}\right\}$ and $\phi_{2}: V\left(T_{p}\langle 1,2,3\rangle \cup\left(\left\lceil\frac{p-3}{2}\right\rceil\right) K_{1}\right) \rightarrow$ $\left\{1,2, \ldots,\left|V\left(T_{p}\langle 1,2,3\rangle\right)\right|+\left\lceil\frac{p-3}{2}\right\rceil\right\}$ have the desired property, for $p \equiv 1(\bmod 4)$ and $p \equiv 0,2,3(\bmod 4)$, respectively. Here, for $p \equiv 1(\bmod 4)$ we label $T_{p}\langle 1,2,3\rangle \cup\left(\frac{p-1}{2}\right) K_{1}$ where $V\left(\left(\frac{p-1}{2}\right) K_{1}\right)=\left\{z_{i}\right\}$ for $1 \leq i \leq \frac{p-1}{2}$ and for $p \equiv 0,2,3(\bmod 4)$, we label $T_{p}\langle 1,2,3\rangle \cup\left\lceil\frac{p-3}{2}\right\rceil K_{1}$ where $V\left(\left\lceil\frac{p-3}{2}\right\rceil K_{1}\right)=\left\{z_{i}\right\}$ for $1 \leq i \leq\left\lceil\frac{p-3}{2}\right\rceil$.

$$
\phi_{1}\left(x_{i}\right)=\phi_{2}\left(x_{i}\right)= \begin{cases}\left\lfloor\frac{3 i-1}{2}\right\rfloor, & \text { if } i \equiv 1,2 \quad(\bmod 4) \\ \left\lfloor\frac{3 i-2}{2}\right\rfloor, & \text { if } i \equiv 0,3 \quad(\bmod 4)\end{cases}
$$

The isolated vertices $z_{i}$, for $1 \leq i \leq \frac{p-1}{2}$ under labeling $\phi_{1}$ and for $1 \leq i \leq\left\lceil\frac{p-3}{2}\right\rceil$ under labeling $\phi_{2}$ are labeled as $\phi_{1}\left(z_{i}\right)=\phi_{2}\left(z_{i}\right)=3 i+1$ for $i$ odd and $\phi_{1}\left(z_{i}\right)=\phi_{2}\left(z_{i}\right)=3 i$ for $i$ even. The edge-sum under the labeling $\phi_{1}$ and $\phi_{2}$ are as follows: 


$$
\begin{aligned}
& w\left(x_{i} x_{i+1}\right)= \begin{cases}\left\lfloor\frac{3 i-1}{2}\right\rfloor+\left\lfloor\frac{3 i+1}{2}\right\rfloor, & \text { if } i \equiv 1,2(\bmod 4) \\
\left\lfloor\frac{3 i-2}{2}\right\rfloor+\left\lfloor\frac{3 i+1}{2}\right\rfloor, & \text { if } i \equiv 3(\bmod 4) \\
\left\lfloor\frac{3 i-2}{2}\right\rfloor+\left\lfloor\frac{3 i+2}{2}\right\rfloor, & \text { if } i \equiv 0(\bmod 4)\end{cases} \\
& w\left(x_{i} x_{i+2}\right)= \begin{cases}\left\lfloor\frac{3 i-1}{2}\right\rfloor+\left\lfloor\frac{3 i+4}{2}\right\rfloor, & \text { if } i \equiv 1,2(\bmod 4) \\
\left\lfloor\frac{3 i-2}{2}\right\rfloor+\left\lfloor\frac{3 i+5}{2}\right\rfloor, & \text { if } i \equiv 0,3(\bmod 4)\end{cases} \\
& w\left(x_{i} x_{i+3}\right)= \begin{cases}\left\lfloor\frac{3 i-1}{2}\right\rfloor+\left\lfloor\frac{3 i+7}{2}\right\rfloor, & \text { if } i \equiv 1(\bmod 4) \\
\left\lfloor\frac{3 i-1}{2}\right\rfloor+\left\lfloor\frac{3 i+8}{2}\right\rfloor, & \text { if } i \equiv 2(\bmod 4) \\
\left\lfloor\frac{3 i-2}{2}\right\rfloor+\left\lfloor\frac{3 i+8}{2}\right\rfloor, & \text { if } i \equiv 3(\bmod 4) \\
\left\lfloor\frac{3 i-2}{2}\right\rfloor+\left\lfloor\frac{3 i+7}{2}\right\rfloor, & \text { if } i \equiv 0(\bmod 4)\end{cases}
\end{aligned}
$$

It is easy to see that the edge-sums form $q$ consecutive integer. Which completes the proof.

From Theorem 2.1, the super edge-magic deficiency of $T_{p}\langle 1,2,3\rangle$ i.e $\mu_{s}\left(T_{p}\langle 1,2,3\rangle\right) \geq$ $\left\lceil\frac{p-3}{2}\right\rceil$, combine with Theorem 2.4, which leads the following corollary and open problem.

2.5. Corollary. Consider the graph $T_{p}\langle 1,2,3\rangle$ with $p$ vertices and $q$ edges. For $p \not \equiv 1$ $(\bmod 4)$, the super edge-magic deficiency for $T_{p}\langle 1,2,3\rangle$ is $\left\lceil\frac{p-3}{2}\right\rceil$ i.e $\mu_{s}\left(T_{p}\langle 1,2,3\rangle\right)=$ $\left\lceil\frac{p-3}{2}\right\rceil$.

2.6. Open Problem. Calculate the super edge-magic deficiency for the Toeplitz graph $T_{p}\langle 1,2,3\rangle$ for $p \equiv 1(\bmod 4)$.

2.7. Theorem. Consider the graph $T_{p}\langle 1,2,3,4\rangle$ with $p$ vertices, $q$ edges, then

$$
p-3 \leq \mu_{s}\left(T_{p}\langle 1,2,3,4\rangle\right) \leq \begin{cases}\left\lceil\frac{p-4}{5}\right\rceil+p-3, & \text { if } p \equiv 3,4 \quad(\bmod 5) \\ \left\lceil\frac{p}{5}\right\rceil+p-3, & \text { if } p \equiv 0,2 \quad(\bmod 5) \\ \left\lceil\frac{p}{5}\right\rceil+p-2, & \text { if } p \equiv 1 \quad(\bmod 5)\end{cases}
$$

Proof. Let $x_{1}, x_{2}, \ldots, x_{p}$ be the vertex sequence of $T_{p}\langle 1,2,3,4\rangle$ and the edges of

$T_{p}\langle 1,2,3,4\rangle$ are $\left\{x_{i} x_{i+1}: 1 \leq i \leq p-1\right\} \cup\left\{x_{i} x_{i+2}: 1 \leq i \leq p-2\right\} \cup\left\{x_{i} x_{i+3}: 1 \leq i \leq\right.$ $p-3\} \cup\left\{x_{i} x_{i+4}: 1 \leq i \leq p-4\right\}$.

From Theorem 2.1 the lower bound for the super edge-magic deficiency of $T_{p}\langle 1,2,3,4\rangle$ is $\mu_{s}\left(T_{p}\langle 1,2,3,4\rangle\right) \geq p-3$. For the upper bound let $p$ be a nonnegative integer. According to Lemma 1.1 it is sufficient to prove that there exists a vertex labeling with the property that the edge-sums under this labeling are consecutive integers. It is easy to see that the following labelings $\phi_{1}: V\left(T_{p}\langle 1,2,3,4\rangle \cup\left(\left\lceil\frac{p-4}{5}\right\rceil+p-3\right) K_{1}\right) \rightarrow$ $\left\{1,2, \ldots,\left|V\left(T_{p}\langle 1,2,3,4\rangle\right)\right|+\left\lceil\frac{p-4}{5}\right\rceil+p-3\right\}, \phi_{2}: V\left(T_{p}\langle 1,2,3,4\rangle \cup\left(\left\lceil\frac{p}{5}\right\rceil+p-3\right) K_{1}\right) \rightarrow$ $\left\{1,2, \ldots,\left|V\left(T_{p}\langle 1,2,3,4\rangle\right)\right|+\left\lceil\frac{p}{5}\right\rceil+p-3\right\}$ and $\phi_{3}: V\left(T_{p}\langle 1,2,3,4\rangle \cup\left(\left\lceil\frac{p}{5}\right\rceil+p-2\right) K_{1}\right) \rightarrow$ $\left\{1,2, \ldots,\left|V\left(T_{p}\langle 1,2,3,4\rangle\right)\right|+\left\lceil\frac{p}{5}\right\rceil+p-2\right\}$ have the desired property, for $p \equiv 3,4(\bmod 5)$, $p \equiv 0,2(\bmod 5)$ and $n \equiv 1(\bmod 5)$, respectively. Here, for $p \equiv 3,4(\bmod 5)$ we label $T_{p}\langle 1,2,3,4\rangle \cup\left(\left\lceil\frac{p-4}{5}\right\rceil+p-3\right) K_{1}$ where $V\left(\left(\left\lceil\frac{p-4}{5}\right\rceil+p-3\right) K_{1}\right)=\left\{z_{i}\right\}$ for $1 \leq i \leq$ $\left\lceil\frac{p-4}{5}\right\rceil+p-3$, for $p \equiv 0,2(\bmod 5)$, we label $T_{p}\langle 1,2,3,4\rangle \cup\left(\left\lceil\frac{p}{5}\right\rceil+p-3\right) K_{1}$ where $\left.V\left(\left\lceil\frac{p}{5}\right\rceil+p-3\right) K_{1}\right)=\left\{z_{i}\right\}$ for $1 \leq i \leq\left\lceil\frac{p}{5}\right\rceil+p-3$ and for $p \equiv 1(\bmod 5)$, we label $T_{p}\langle 1,2,3,4\rangle \cup\left(\left\lceil\frac{p}{5}\right\rceil+p-2\right) K_{1}$ where $\left.V\left(\left\lceil\frac{p}{5}\right\rceil+p-2\right) K_{1}\right)=\left\{z_{i}\right\}$ for $1 \leq i \leq\left\lceil\frac{p}{5}\right\rceil+p-2$.

$$
\phi_{1}\left(x_{i}\right)=\phi_{2}\left(x_{i}\right)=\phi_{3}\left(x_{i}\right)=\left\{\begin{array}{lll}
2 i-1+\left\lceil\frac{i-1}{5}\right\rceil, & \text { if } i \equiv 1 & (\bmod 5) \\
2 i-2+\left\lceil\frac{i-2}{5}\right\rceil, & \text { if } i \equiv 2 & (\bmod 5) \\
2 i-3+\left\lceil\frac{i-3}{5}\right\rceil, & \text { if } i \equiv 3 & (\bmod 5) \\
2 i-4+\left\lceil\frac{i}{5}\right\rceil, & \text { if } i \equiv 4 & (\bmod 5) \\
2 i-3+\left\lceil\frac{i}{5}\right\rceil, & \text { if } i \equiv 0 & (\bmod 5)
\end{array}\right.
$$


The isolated vertices $z_{i}$, for $1 \leq i \leq\left\lceil\frac{p-4}{5}\right\rceil+p-3, p \equiv 3,4(\bmod 5)$ under labeling $\phi_{1}$, for $1 \leq i \leq\left\lceil\frac{p}{5}\right\rceil+p-3$, for $p \equiv 0,2(\bmod 5)$ under labeling $\phi_{2}$ and for $1 \leq i \leq\left\lceil\frac{p}{5}\right\rceil+p-2$ for $p \equiv 1(\bmod 5)$ under labeling $\phi_{3}$ are labeled as:

$$
\phi_{1}\left(z_{i}\right)=\phi_{2}\left(z_{i}\right)=\phi_{3}\left(z_{i}\right)=\left\{\begin{array}{lll}
i+2+\left\lceil\frac{5 i}{6}\right\rceil, & \text { if } i \equiv 1,2 & (\bmod 6) \\
i+1+\left\lceil\frac{5 i}{6}\right\rceil, & \text { if } i \equiv 3,4 & (\bmod 6) \\
i+\left\lceil\frac{5 i}{6}\right\rceil, & \text { if } i \equiv 0,5 & (\bmod 6)
\end{array}\right.
$$

It is easy to see that the edge-sums form $q$ consecutive integer. (See Figure 2 for illustration.) Therefore by using Lemma $1.1, \phi_{1}, \phi_{2}, \phi_{3}$ can be extended to a super edge-magic labeling. It follows that

$$
\mu_{s}\left(T_{p}\langle 1,2,3,4\rangle\right) \leq \begin{cases}\left\lceil\frac{p-4}{5}\right\rceil+p-3, & \text { if } p \equiv 3,4 \quad(\bmod 5) \\ \left\lceil\frac{p}{5}\right\rceil+p-3, & \text { if } p \equiv 0,2 \quad(\bmod 5) \\ \left\lceil\frac{p}{5}\right\rceil+p-2, & \text { if } p \equiv 1 \quad(\bmod 5)\end{cases}
$$

Combining with lower bound, which completes the proof.

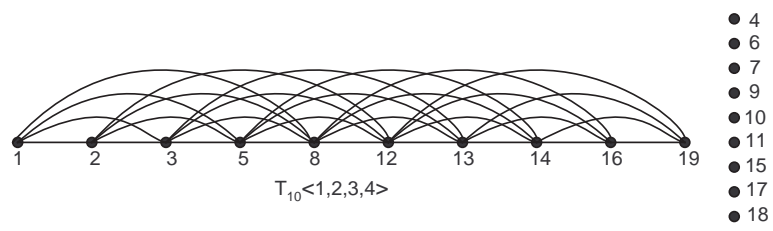

Figure 2. An illustration for the labeling given in the proof of Theorem 2.7.

2.8. Open Problem. Improve, if possible, the bounds for the super edge-magic deficiency for the Toeplitz graph $T_{p}\langle 1,2,3,4\rangle$ or show that the bounds provided are sharp.

\section{Closing Remarks}

We have studied that the lower bounds for the super edge-magic deficiency of the Toeplitz graph $T_{p}\left\langle t_{1}, \ldots, t_{k}\right\rangle$. For $t$ even we determined that the super edge-magic deficiency of Toeplitz graph $\mu_{s}\left(T_{p}\langle 1, t\rangle\right)=0$. We also studied the upper bounds for the super edge-magic deficiency for Toeplitz graph $T_{p}\langle 1,2,3\rangle$ and $T_{p}\langle 1,2,3,4\rangle$. It would be interesting to find the super edge-magic deficiency of $\left.T_{p}\langle 1, t\rangle\right)$ for $t$ odd. We encourage researchers to try to determine the super edge-magic deficiency of other graphs for further research. In fact, it seems to be a very challenging problem to find the exact value for the super edge-magic deficiency of families of graphs.

Acknowledgement:

The authors wish to thank the anonymous referee for his/her valuable comments. We also thank the Deanship of Research, Jazan University for supporting this project under the Grant No. 000114/7/37.

\section{References}

[1] B. D. Acharya, S. M. Hegde, Strongly indexable graphs, Discrete Math., 93 (1991), 123-129.

[2] A. Ahmad, I. Javaid, M. F. Nadeem and R. Hasni, On Super edge-magic deficiency of some families related to ladder graphs, Australas. J. Combin., 51, 201-208, 2011. 
[3] A. Ahmad, M. Bača, M. F. Nadeem, On edge irregularity strength of Toeplitz graphs, U.P.B. Sci. Bull., Series A, 78(4), 155-162, 2016.

[4] M. Bača, Y. Bashir, M. F. Nadeem, A. Shabbir, On super edge-antimagic total labeling of Toeplitz graphs, Springer Proceedings in Mathematics 83 Statistics, 98 (2015), 1-11.

[5] H. Enomoto, A. Lladó, T. Nakamigawa, and G. Ringel, Super edge-magic graphs, SUT J. Math., 34, 105-109, 1998.

[6] R. M. Figueroa-Centeno, R. Ichishima and F.A. Muntaner-Batle, The place of super edgemagic labeling among other classes of labeling, Discrete Math., 231, 153-168, 2001.

[7] R. M. Figueroa-Centeno, R. Ichishima and F.A. Muntaner-Batle, On the super edge-magic deficiency of graphs, Electron. Notes Discrete Math., 11, 2002.

[8] R. M. Figueroa-Centeno, R. Ichishima and F.A. Muntaner-Batle, On the super edge-magic deficiency of graphs, Ars Combin., 78, 2006.

[9] A. Kotzig and A. Rosa, Magic valuaton of finite graphs, Canad. Math. Bull., 13(4), 451-461, 1970.

[10] A. A. G. Ngurah, R. Simanjuntak and E.T. Baskoro, On the super edge-magic deficiencies of graphs, Australas. J. Combin., 40, 3-14, 2008.

[11] J.Y. Park, J.H. Choi and J.H. Bae, On super edge-magic labeling of some graphs, Bull. Korean Math. Soc., 45, No.1, 11-21, 2008. 